\title{
Long-term treatment with metformin in the prevention of fatty liver in Zucker diabetic fatty rats
}

\author{
Yi Sui ${ }^{{ }^{*}+}$, Xianhe Kong ${ }^{2 \dagger}$, Rongrong Fan ${ }^{3}$, Yanbin Ye ${ }^{1}$, Haiyan Mai ${ }^{1}$, Shuyu Zhuo ${ }^{1}$, Wei Lu', Peishan Ruan ${ }^{1}$, \\ Shi Fang ${ }^{1 *}$ and Tao Yang ${ }^{4^{*}}$
}

\begin{abstract}
Background: Treatment with metformin, the biguanide of hepatic insulin sensitizer, in patients with non-alcoholic fatty liver disease (NAFLD) has been reported with contradictory findings regarding the effectiveness on blood lipids and liver histology. In this study, we aimed to explore the preventive effects of metformin on NAFLD in Zucker diabetic fatty (ZDF) rats.

Methods: Male ZDF rats and Zucker lean rats aged 4-8 weeks were subjected to vehicle or metformin treatment for 6 months. Liver cDNA microarray assay, and protein semiquantitative and histological examinations were performed.

Results: Data demonstrated that ZDF rats developed hyperglycemia, hyperlipidemia, liver deficiency and hepatocyte degeneration. The metformin treatment significantly reduced post-load blood glucose levels, but not blood lipid profiles or liver enzyme levels. Hepatocyte degeneration was not attenuated after the treatment. The metformin-treated ZDF rats showed activation of AMP-activated protein kinase by Western blot and overexpression of cytochrome $\mathrm{C}$ oxidase by immunofluorescent microscopy. Gene expression microarray assay demonstrated that a panel of genes participating in glucose and lipid metabolisms were changed in the ZDF rats, and most of the altered genes involved in glucose and cholesterol metabolisms, but not those in fatty acid metabolisms, were corrected by the metformin treatment. No genes associated with inflammation, apoptosis, fibrosis, or cell death were overexpressed in the metformin-treated ZDF rats.
\end{abstract}

Conclusions: These results suggest that long-term metformin treatment presents no preventive effect for NAFLD in ZDF rats.

Keywords: Metformin, Non-alcoholic fatty liver disease, Zucker diabetic fatty rats, Gene expression profiling

\section{Background}

Metformin, a biguanide that improves hepatic insulin resistance and hyperglycemia, is the first-line therapeutic agent for patients with type 2 diabetes, especially those who also have hyperlipidemia and obesity $[1,2]$. Since

\footnotetext{
*Correspondence: suiyi@mail.sysu.edu.cn; 1343439560@qq.com; mryangtao2005@yahoo.com

${ }^{\dagger}$ Yi Sui and Xian-He Kong contributed equally to this work

${ }^{1}$ Department of Clinical Nutrition, The First Affiliated Hospital, Sun Yat-sen

University, Guangzhou 510080, China

${ }^{4}$ Center for Medical Research and Innovation, Shanghai Pudong Hospital,

Fudan University Pudong Medical Center, Shanghai 201399, China
}

Full list of author information is available at the end of the article it was noted that some patients with fatty liver diseases had improved liver function tests after metformin treatment, metformin was studied as a candidate for the treatment of non-alcoholic fatty liver disease (NAFLD) [3-6]. Short-term treatment with metformin has frequently been reported with beneficial effects on lowering blood lipid levels and protecting hepatocytes from lipid accumulation [2, 7-9], but several studies with long-term metformin treatment did not show hepatic histological protection [6, 10-12]. Paradoxically, metformin might induce hepatotoxicity including acute hepatitis [13-16] and cholestasis [17]. In all the cases, several weeks of metformin treatment induced elevated hepatic enzyme 
concentrations. These adverse effects resolved after discontinuation of metformin treatment. The mechanisms underlying the lipid-lowering effects and hepatotoxicity are still unclear.

The primary purpose of present study was to test the efficacy of metformin on the prevention of fatty liver disease in Zucker diabetic fatty (ZDF) rats. Adult ZDF rats develop hyperlipidemia, hyperglycemia, hyperinsulinemia, insulin resistance, fatty liver and hepatocyte degeneration owing to an inherited mutation of the leptin receptor. Hereby, young ZDF rats were treated with metformin for 6 months, followed by mRNA liver tissue array to investigate the long-term effects of metformin treatment on the development of hyperlipidemia and fatty liver disease.

\section{Materials and methods Rat experiments}

Zucker fatty rats were obtained from Beijing Vital River Laboratory Animal Co., Ltd. (Beijing, China) and introduced into the Laboratory Animal Services Centre at the Fudan University Pudong Medical Center. The animals were caged in pairs, housed at $23 \pm 1{ }^{\circ} \mathrm{C}$ with a 12 -h dark/light cycle, having free access to water and fed on a standard laboratory rat diet (5001 Rodent Diet, LabDiet, St Louis, MO). Ethical approval for animal studies was according to the Animal Experimentation Ethics Committee of Fudan University Pudong Medical Center.

Fourteen male ZDF rats and six Zucker lean rats aged 4-8 weeks were divided into three groups: (1) ZDF rats treated with metformin $(50 \mathrm{mg} / \mathrm{kg}$ body weight, $\mathrm{n}=7)$, (2) ZDF rats treated with vehicle of distilled water $(10 \mathrm{~mL} / \mathrm{kg}, n=7)$, and (3) Zucker lean rats treated with vehicle of distilled water $(10 \mathrm{~mL} / \mathrm{kg}, n=6)$. All treatments were conducted through oral administration once daily. After the 6-month treatment, rats were killed at fasting state. Liver tissues were collected freshly for cDNA microarray or fixed in 10\% neutral formaldehyde for histological analysis.

Body weight was monitored before rats were killed; oral glucose tolerance tests (OGTT) were conducted by gavage of dextrose $(2.5 \mathrm{~g} / \mathrm{kg}$ body weight) following $8 \mathrm{~h}$ of fasting. Blood from the tail vein was used for measuring blood glucose levels with a blood glucose meter (Onetouch Ultra, LifeScan, Inc.; US). Fasting blood samples were taken for the measurement of blood lipids and liver functions.

Total RNA isolation and reverse transcription

Total RNA from rat livers was extracted using RNeasy Mini Kit with DNase digestion option (Qiagen, Valencia, CA) to remove residual genomic DNA according to the manufacturer's instructions. Total RNA prepared from individual animals was pooled together using equal amount of total RNA from individual animals in each group. The total RNA samples were sent to Li Ka Shing Institute of Health Sciences Core Lab (The Chinese University of Hong Kong, Hong Kong) for reverse transcription, labeling, microarray hybridization, washing, and scanning using the Affymetrix GeneChip ${ }^{\circledR}$ Gene 1.0 ST Array System. Briefly, 100 ng of the pooled total RNA was used as a template for cDNA synthesis and subsequent labeling using the GeneChip ${ }^{\circledR}$ Whole Transcript Sense Target Labeling Assay from Affymetrix (Affymetrix, Santa Clara, CA). The generation of hybridization cocktails, hybridization to DNA microarrays, and fluorescent labeling of RNA targets were carried out as described in the standard Affymetrix protocol. Fluorescence-labeled microarrays were scanned with GeneChip ${ }^{\circledR}$ Scanner 3000 (Affymetrix). Results were analyzed with GeneSpring GX 10.0 software with global normalization and fold change $\geq 2.0$ was chosen as the cut-off of different expression levels of the compared genes.

\section{Real-time quantitative PCR}

The mRNA samples were estimated via quantification of relative expression levels using TaqMan 2X Universal PCR Master Mix (Applied Biosystems Inc, CA) according to the manufacturer's protocols. For mRNA amplification, primers were synthesized by Invitrogen (Invitrogen, Cergy-Pontoise, France) as listed in Table 1. Real-time PCR was performed under the following conditions: $95^{\circ} \mathrm{C}$, $10 \mathrm{~min} ; 95^{\circ} \mathrm{C}, 15 \mathrm{~s}$ followed by $60^{\circ} \mathrm{C}, 60 \mathrm{~s}$ and 40 times of cycles. PCR amplifications were performed on a $7900 \mathrm{HT}$ Fast real-time PCR system and data were analyzed with the 7900HT Fast system SDS software v2.0 (Applied Biosystems). Relative mRNA expression was quantified by the comparative $\mathrm{Ct}$ method and expressed as $2^{-\Delta \Delta \mathrm{Ct}}$.

Table 1 Primer sequences for real-time PCR

\begin{tabular}{lll}
\hline Gene name & \multicolumn{2}{l}{ Primer sequences } \\
\hline G6pdx & Forward & 5'-GAC TGT GGG CAA GCT CCT CAA-3' \\
& Reverse & 5'-GCT AGT GTG GCT ATG GGC AGG T-3' \\
Hmgcs1 & Forward & 5'-TCA AGG CTT GAC TCA AGA ACG-3' \\
& Reverse & 5'-GGA ATA TGC TCT GTA GCT GTG-3' \\
Cyp7a1 & Forward & 5'-ATG ACA CGC TCT CCA CCT TTG A-3' \\
& Reverse & 5'-AGC TCT TGG CCA GCA CTC TGT-3' \\
Igfbp1 & Forward & 5'-GAA GCT TTT CTC ATC TCC ATA CAT GT-3' \\
& Reverse & 5'-AAG GCC CCT ACC TCA GAC TGA-3' \\
Scd-1 & Forward & 5'-GCTTGT GGA GCC ACA GGA CT-3' \\
& Reverse & 5'-ATC CCG GGC CCA TTC ATA-3' \\
\end{tabular}




\section{Western blot}

Total protein extracts were prepared from frozen liver tissues. Briefly, tissue was homogenized in a RIPA buffer and protein extracts $(100 \mu \mathrm{g})$ were separated by SDSPAGE using $10 \%$ gels. The resolved proteins were then transferred onto nitrocellulose membranes and incubated with primary antibodies of rabbit anti-AMPK $\alpha$ (1:1000, Cell Signaling Technology, Danvers, MA), rabbit anti-p-AMPK (1:1000, Cell Signaling Technology), and rabbit anti- $\beta$-actin (1:10,000, Abcam, Cambridge, MA) overnight at $4{ }^{\circ} \mathrm{C}$. After washing with TBS-T, membranes were incubated with anti-rabbit secondary antibody conjugated to horseradish peroxidase (Upstate, Temecula, MA) with dilution of 1:5000. Protein bands were detected by enhanced chemiluminescence reagent (Amersham, Piscataway, NJ) and then exposed to Hyperfilm. The major protein bands detected were approximately $62 \mathrm{KD}$ for AMPK and p-AMPK, $47 \mathrm{KD}$ for $\beta$-actin. Signals were then quantitated by densitometry and corrected for the $\beta$-actin signal.

\section{Histopathological examination}

Liver tissues fixed in neutral formaldehyde were embedded in paraffin. Sections $(4 \mu \mathrm{m})$ were stained with periodic acid-Schiff (PAS) for routine structural examination. For immunofluorescence examination, slides were stained with antibody of mouse anti-cytochrome c oxidase (CCO, 1:200, Invitrogen, Cergy-Pontoise, France) to evaluate the mitochondria function and counterstained with DAPI. Stained slides were examined with a Zeiss Axioplan 2 imaging microscope (Carl Zeiss, Hamburg, Germany), and representative images were captured using a SPOT digital camera (Diagnostic Instruments Inc, Sterling Heights, MI).

\section{Statistical analysis}

Data are expressed as mean \pm standard deviation. SPSS 16.0 (Statistics Package for the Social Sciences 16.0,
Chicago, IL) was used to perform statistical analysis. One-way ANOVA was adopted to analyze the statistical differences of biochemical parameters among all groups, and Bonferroni test was used for post hoc multiple comparisons between different groups. A two-tailed $p$ value of less than 0.05 was taken as the criterion for a statistically significant difference.

\section{Results}

\section{Biochemical and metabolic profiles}

During this study of 6 months, the ZDF rats showed progressive body weight gain compared with age-matched Zucker lean rats (Table 2). The weight gain in ZDF rats was not affected by the 6-month metformin treatment. Metformin did not significantly change blood total cholesterol and triglyceride in ZDF rats. However, treatment with metformin indeed significantly reduced the 2-h OGTT blood glucose levels.

ZDF rats developed liver deficiency at the age of 8 months after the 6-month vehicle treatment, as reflected by decreased serum albumin level and elevated serum alanine aminotransferase (ALT) and aspartate transaminase (AST) levels. Compared with the vehicle, metformin treatment showed a trend of moderating the increase of liver enzyme levels, but this trend was not statistically significant (Table 2).

\section{Histological assessment of liver}

PAS staining and light microscopy were performed to illustrate the morphological changes of liver histology. The untreated ZDF rats showed patches of ballooning degeneration and fatty change with cytoplasmic microvacuolation in hepatocytes (Fig. 1). These changes were even more obvious in metformin-treated ZDF rats, in which patches of the degenerated cells were surrounded with condensed hepatocytes.

Table 2 Metabolic characters of rats after 6-month treatment

\begin{tabular}{lccc}
\hline & Zucker lean, $\boldsymbol{n = 6}$ & ZDF + Met, $\boldsymbol{n = 7}$ & ZDF+V, $\mathbf{n = 7}$ \\
\hline Body weight (g) & $390 \pm 12$ & $491 \pm 29^{\mathrm{a}}$ & $485 \pm 49^{\mathrm{b}}$ \\
2-h OGTT blood glucose (mmol/L) & $7.9 \pm 1.7$ & $11.4 \pm 2.3^{\mathrm{ac}}$ & $14.7 \pm 2.3^{\mathrm{b}}$ \\
Serum total cholesterol (mmol/L) & $1.07 \pm 0.07$ & $4.9 \pm 1.0^{\mathrm{a}}$ & $5.0 \pm 0.6^{\mathrm{b}}$ \\
Serum triglyceride (mmol/L) & $0.32 \pm 0.02$ & $8.9 \pm 3.3^{\mathrm{a}}$ & $8.7 \pm 1.9^{\mathrm{b}}$ \\
Serum total protein (g/L) & $70.0 \pm 1.7$ & $70.6 \pm 1.7$ & $73.0 \pm 2.3$ \\
Serum albumin (g/L) & $20.7 \pm 0.6$ & $16.1 \pm 2.0^{\mathrm{a}}$ & $15.7 \pm 1.4^{\mathrm{b}}$ \\
ALT (U/L) & $79.3 \pm 11.4$ & $139.9 \pm 25.9^{\mathrm{a}}$ & $155.7 \pm 79.2^{\mathrm{b}}$ \\
AST (U/L) & $86.7 \pm 6.1$ & $120.6 \pm 22.8^{\mathrm{a}}$ & $156.3 \pm 61.0^{\mathrm{b}}$ \\
\hline
\end{tabular}

Data are mean \pm SD

OGTT oral glucose tolerance test, ALT alanine aminotransferase, AST aspartate transaminase

a,b $p<0.05$ versus Zucker lean, ${ }^{c} p<0.05$ versus $V$ 


\section{AMPK activation and cytochrome c oxidase (CCO) overexpression}

Based on the previous study which demonstrated metformin lowered blood glucose and lipids by activating AMP-activated protein kinase (AMPK) [18], protein expression level of AMPK was semi-quantitatively measured by Western blot. Increased protein levels of total AMPK but not phosphorylated-AMPK (p-AMPK) were found in vehicle-treated rats compared with that in Zucker lean rats. Metformin treatment increased p-AMPK but not the total AMPK protein level compared with those in vehicle-treated rats (Fig. 2). The higher ratio of $\mathrm{p}$-AMPK to total AMPK level implied the activation of AMPK in metformin-treated ZDF rats.

Considering the important roles of $\mathrm{CCO}$ in the processes of glucose and lipid catabolisms in mitochondria, protein expression level of CCO was analyzed by immunofluorescence microscopy. There was a homogeneously weak staining of $\mathrm{CCO}$ in the normal liver of Zucker lean rats (Fig. 3). The vehicle-treated ZDF rats showed scattered or stripe-like distribution of positive labeling, while the metformin-treated rats demonstrated patches of strong cytoplasmic staining of CCO. In metformintreated rats, the positively stained hepatocytes were usually found adjacent to degenerated hepatocytes which often exhibited weak positivity. Occasionally, edges of the cytoplasmic vacuoles were strongly stained with $\mathrm{CCO}$ antibody.

\section{Global profiling of mRNA expression after metformin treatment}

The activity of hepatocyte in substrate catabolic processes as reflected by AMPK activation and $\mathrm{CCO}$ overexpression in the metformin-treated ZDF rats was not consistent with the observation that metformin did not

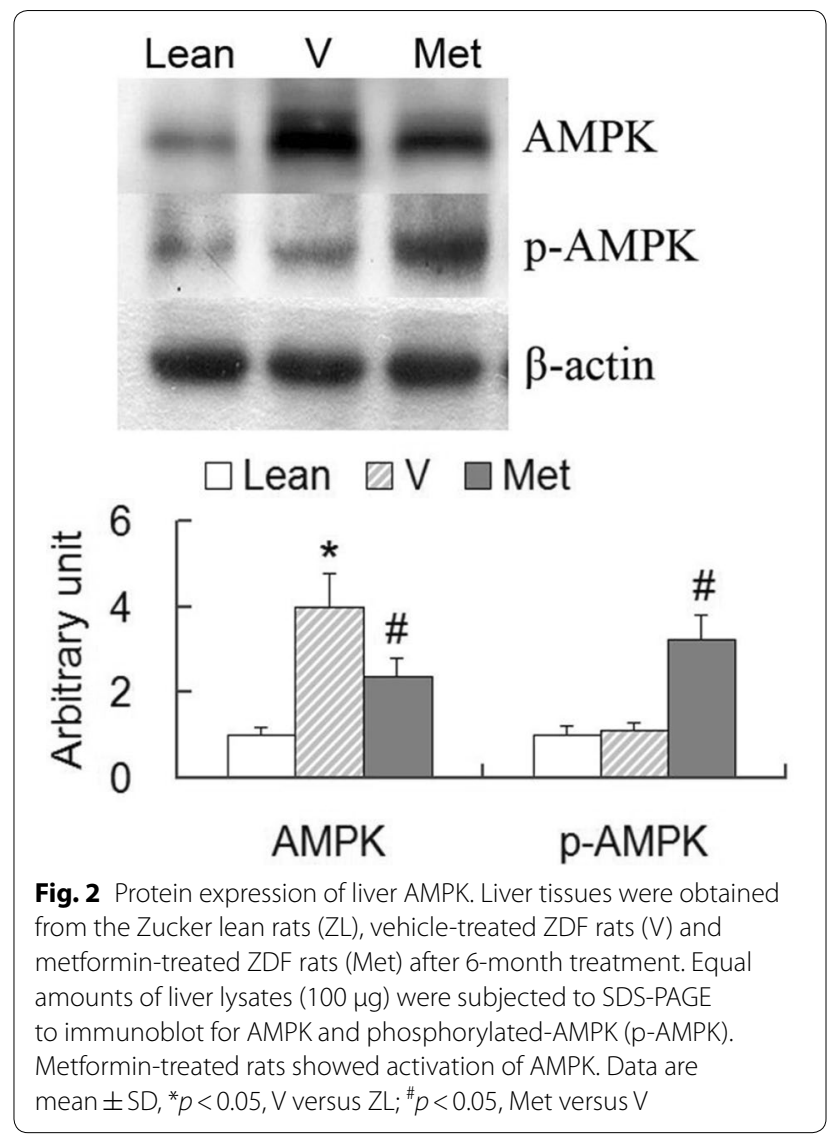

attenuate blood lipids and fatty liver. To elucidate the impact of long-term metformin treatment on global gene expressions, we examined the profiling of mRNA expression levels using microarray technology. Microarray analysis detected 22,342 liver genes in total, while metformin treatment altered the expression levels of 92 genes on the
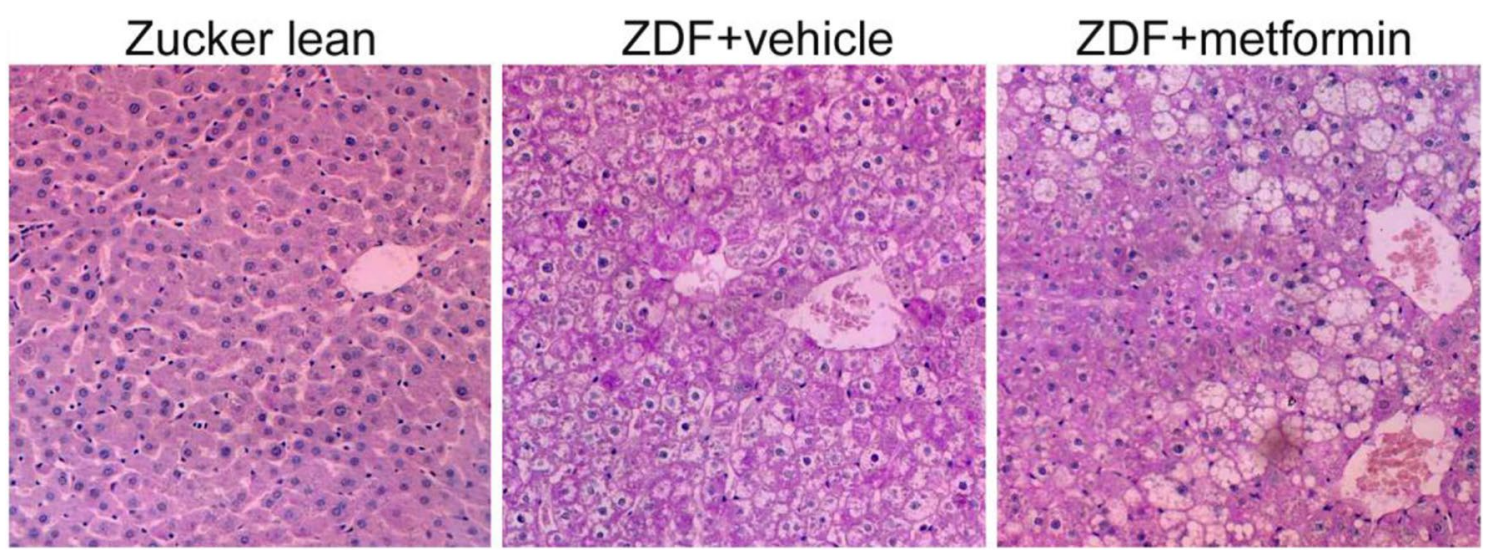

Fig. 1 Ballooning degeneration and fatty change of hepatocytes in Zucker rats. Liver tissues were collected after 6-month treatment with vehicle or metformin. PAS staining demonstrated patch-distributed ballooning degeneration and fatty change of hepatocytes in vehicle- and metformin-treated ZDF rats 

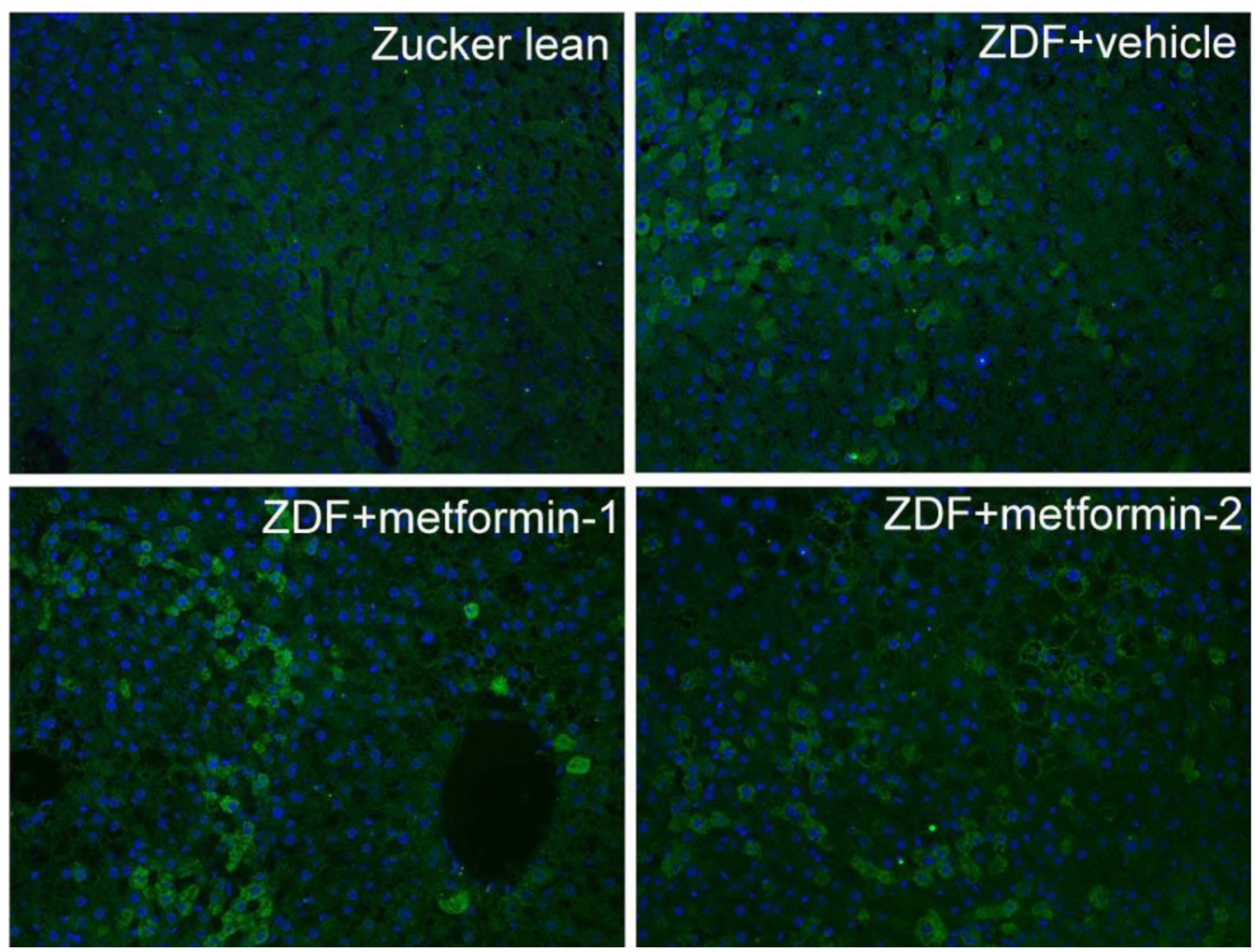

Fig. 3 Immunofluorescence microscopy of cytochrome c oxidase (CCO). Liver tissues obtained after 6-month treatment with vehicle or metformin were stained with anti-CCO (green). The CCO staining was weak and uniform in Zucker lean rats, whereas Zucker diabetic fatty rats showed patched of strong CCO reactivity. Stronger CCO labeling was seen at the intracellular vacuoles in hepatocytes with ballooning degeneration from metformin-treated rats (ZDF + metformin-1, (ZDF + metformin-2)

criteria of fold change $\geq 2.0$ when compared with those of the untreated ZDF rats. Among the 92 genes, 39 genes (42.4\%) were involved in metabolic processes, including 16 genes in lipid metabolism, 11 in sterol/steroid metabolism, 3 in fatty acid metabolism, 7 in glucose metabolism, and 4 genes in bile acid metabolism.

We further examined the genes amended by the metformin treatment. In all the detected genes, 66 genes were significantly altered by the gene mutation of leptin receptor in the ZDF rats compared with Zucker lean rats on the criteria of fold change $\geq 2.0$ (Table 3 ). In the annotated 55 genes, most of them were related to the metabolic processes, including 12 genes in lipid metabolism, 5 in sterol/steroid metabolism, 4 in fatty acid metabolism, 4 in glucose metabolism, and 3 in bile acid metabolism. However, none of the genes in insulin signaling pathway such as insulin receptor, insulin receptor substrates and glucose transporters were affected by the leptin receptor mutation in ZDF rats even when we reduced the fold change criterion to 1.5 . Among the 55 significantly altered genes, $36(65 \%)$ genes were corrected by metformin treatment (Table 3), including $6(50 \%)$ genes in lipid metabolism, $4(80 \%)$ in sterol/steroid metabolism, 3 (75\%) genes in glucose metabolism, and 2 (67\%) genes in bile acid metabolism. Remarkably, all the six corrected genes in lipid metabolisms were related to cholesterol metabolism, while none of the genes were related to fatty acid metabolism (Table 3).

In the amended genes, the most significantly altered gene is insulin-like growth factor-binding protein-1 (IGFBP1), a growth-related gene. IGFBP1 was downregulated by 5.3 -fold by the leptin receptor gene mutation in the vehicle-treated ZDF rats and up-regulated by 19.3-fold by metformin treatment. Another important gene corrected by the metformin treatment was Cyp7a1 (cytochrome P450, family 7, subfamily a, polypeptide 1 ), the key enzyme in bile acid synthetic process for the removal of cholesterol from the liver in the form of bile acid. As shown in Table 3, the metformin treatment also corrected other genes encoding the key enzymes in multiple metabolic processes, such as glucose-6-phosphate dehydrogenase (G6pdx, the rate-limiting enzyme of the pentose phosphate pathway in maintenance of NADPH), 
Table 3 Genes modified by metformin treatment

\begin{tabular}{|c|c|c|c|c|c|}
\hline Gene symbol & V vs. ZL & & Met vs. V & & Gene description \\
\hline \multicolumn{6}{|c|}{ Genes corrected by metformin } \\
\hline $\operatorname{lgfbp} 1^{9}$ & 5.25 & $\downarrow$ & 19.28 & $\uparrow$ & Insulin-like growth factor-binding protein 1 \\
\hline Ccrn4lb & 4.03 & $\downarrow$ & 10.24 & $\uparrow$ & CCR4 carbon catabolite repression 4-like B \\
\hline Stac3 & 12.77 & $\downarrow$ & 9.35 & $\uparrow$ & SH3- and cysteine-rich domain 3 \\
\hline $\mathrm{Sds} s^{9}$ & 4.83 & $\downarrow$ & 8.56 & $\uparrow$ & Serine dehydratase \\
\hline Pnpla3 & 13.03 & $\uparrow$ & 8.23 & $\downarrow$ & Patatin-like phospholipase domain containing 3 \\
\hline Len2 & 19.50 & $\uparrow$ & 7.81 & $\downarrow$ & Lipocalin 2 \\
\hline Btg2 & 2.09 & $\downarrow$ & 6.96 & $\uparrow$ & B-cell translocation gene 2 , anti-proliferative \\
\hline Coq10b & 2.34 & $\downarrow$ & 5.41 & $\uparrow$ & Coenzyme Q10 homolog B \\
\hline$A 2 m$ & 6.91 & $\uparrow$ & 5.40 & $\downarrow$ & Alpha-2-macroglobulin \\
\hline Cyp7a1 bcs & 2.81 & $\downarrow$ & 5.01 & $\uparrow$ & Cytochrome P450, family 7 , subfamily a, polypeptide 1 \\
\hline Cdh17 & 8.39 & $\downarrow$ & 4.66 & $\uparrow$ & Cadherin 17 \\
\hline LOC313220 & 5.07 & $\downarrow$ & 4.21 & $\uparrow$ & Similar to bile acid coenzyme A \\
\hline Sqle & 2.25 & $\uparrow$ & 4.13 & $\downarrow$ & Squalene epoxidase \\
\hline Me1 & 2.55 & $\uparrow$ & 4.12 & $\downarrow$ & Malic enzyme 1, NADP(+) dependent, cytosolic \\
\hline G6pdx $x^{9}$ & 3.01 & $\uparrow$ & 3.81 & $\downarrow$ & Glucose-6-phosphate dehydrogenase X-linked \\
\hline Rhbdd2 & 2.51 & $\downarrow$ & 3.77 & $\uparrow$ & Rhomboid domain containing 2 \\
\hline $\mathrm{Cml} 4$ & 4.49 & $\downarrow$ & 3.48 & $\uparrow$ & Camello-like 4 \\
\hline Car3 & 4.71 & $\downarrow$ & 3.30 & $\uparrow$ & Carbonic anhydrase 3 \\
\hline Inmt & 8.28 & $\downarrow$ & 3.28 & $\uparrow$ & Indolethylamine $\mathrm{N}$-methyltransferase \\
\hline Nrep & 2.78 & $\downarrow$ & 3.08 & $\uparrow$ & Neuronal regeneration-related protein \\
\hline $\mathrm{Hmgcs} 1^{\mathrm{cs}}$ & 2.00 & $\uparrow$ & 3.07 & $\downarrow$ & 3-Hydroxy-3-methylglutaryl-coenzyme A synthase 1 \\
\hline Mup5 & 3.18 & $\downarrow$ & 3.05 & $\uparrow$ & Major urinary protein 5 \\
\hline RGD1565709 & 2.38 & $\downarrow$ & 3.03 & $\uparrow$ & Similar to ovostatin-2 \\
\hline RGD1562060 & 3.43 & $\uparrow$ & 2.92 & $\downarrow$ & Similar to short-chain dehydrogenase reductase 9 \\
\hline Ddhd1 & 2.84 & $\uparrow$ & 2.89 & $\downarrow$ & DDHD domain containing 1 \\
\hline Por & 2.19 & $\downarrow$ & 2.78 & $\uparrow$ & P450 (cytochrome) oxidoreductase \\
\hline Сyp3a9s & 3.55 & $\downarrow$ & 2.69 & $\uparrow$ & Cytochrome P450, family 3, subfamily a, polypeptide 9 \\
\hline Slc1a2 & 2.64 & $\downarrow$ & 2.46 & $\uparrow$ & Solute carrier family 1, member 2 \\
\hline Fmo1 & 3.91 & $\downarrow$ & 2.45 & $\uparrow$ & Flavin-containing monooxygenase 1 \\
\hline Dhrs7 & 3.59 & $\downarrow$ & 2.39 & $\uparrow$ & Dehydrogenase/reductase (SDR family) member 7 \\
\hline Aox3 & 3.22 & $\downarrow$ & 2.27 & $\uparrow$ & Aldehyde oxidase 3 \\
\hline LOC360228 & 4.29 & $\uparrow$ & 2.25 & $\downarrow$ & WDNM1 homolog \\
\hline Rdh2 & 2.99 & $\downarrow$ & 2.17 & $\uparrow$ & Retinol dehydrogenase 2 \\
\hline $\mathrm{HaO} 2$ & 2.73 & $\downarrow$ & 2.13 & $\uparrow$ & Hydroxyacid oxidase 2 (long chain) \\
\hline Nox4 & 3.57 & $\downarrow$ & 2.07 & $\uparrow$ & NADPH oxidase 4 \\
\hline Obp3/Mup4 & 2.16 & $\downarrow$ & 2.00 & $\uparrow$ & Alpha-2u globulin PGCL4/major urinary protein 4 \\
\hline \multicolumn{6}{|c|}{ Genes uncorrected by metformin } \\
\hline $\mathrm{Dbp}$ & 2.35 & $\downarrow$ & 1.50 & $\uparrow$ & D site albumin promoter-binding protein \\
\hline $\operatorname{Acsm}^{f}$ & 2.72 & $\downarrow$ & 1.82 & $\uparrow$ & Acyl-CoA synthetase medium-chain family member 3 \\
\hline Chka & 2.06 & $\uparrow$ & 1.80 & $\downarrow$ & Choline kinase alpha \\
\hline $\mathrm{Scd} 1^{\mathrm{f}}$ & 2.02 & $\uparrow$ & 1.47 & $\downarrow$ & Stearoyl-coenzyme A desaturase 1 \\
\hline $\operatorname{Ugp} 2^{g}$ & 2.16 & $\downarrow$ & 1.23 & $\uparrow$ & UDP-glucose pyrophosphorylase 2 \\
\hline Inhba & 2.25 & $\uparrow$ & 1.56 & $\downarrow$ & Inhibin beta-A \\
\hline Prtfdc1 & 2.17 & $\downarrow$ & 1.61 & $\uparrow$ & Phosphoribosyl transferase domain containing 1 \\
\hline Es22 & 2.00 & $\downarrow$ & 1.30 & $\uparrow$ & Esterase 22 \\
\hline Ces3 & 2.66 & $\downarrow$ & 1.52 & $\uparrow$ & Carboxylesterase 3 \\
\hline Esm1 & 2.20 & $\uparrow$ & 1.74 & $\downarrow$ & Endothelial cell-specific molecule 1 \\
\hline Prlr & 2.53 & $\uparrow$ & 1.63 & $\downarrow$ & Prolactin receptor \\
\hline
\end{tabular}


Table 3 (continued)

\begin{tabular}{|c|c|c|c|c|c|}
\hline Gene symbol & V vs. ZL & & Met vs & & Gene description \\
\hline $\mathrm{Fabp} 7^{f}$ & 2.14 & $\downarrow$ & 1.63 & $\uparrow$ & Fatty acid-binding protein 7 , brain \\
\hline Slc16a10 & 2.10 & $\downarrow$ & 1.77 & $\uparrow$ & Solute carrier family 16 , member 10 \\
\hline Upp2 & 3.33 & $\downarrow$ & 1.95 & $\uparrow$ & Uridine phosphorylase 2 \\
\hline $\operatorname{lnsig} 1^{c s}$ & 3.06 & $\uparrow$ & 1.54 & $\downarrow$ & Insulin-induced gene 1 \\
\hline RGD1561619 & 2.83 & $\downarrow$ & 1.79 & $\uparrow$ & Similar to Camello-like 2 \\
\hline Slco1a4 & 2.20 & $\downarrow$ & 1.23 & $\uparrow$ & Solute carrier organic anion transporter family, member 1a4 \\
\hline Adfp $^{f}$ & 2.06 & $\uparrow$ & 1.25 & $\downarrow$ & Adipose differentiation-related protein \\
\hline Ces3 & 2.50 & $\downarrow$ & 1.62 & $\uparrow$ & Carboxylesterase 3 \\
\hline
\end{tabular}

${ }^{\mathrm{b}}$ Bile acid metabolisms, ${ }^{\mathrm{c}}$ cholesterol metabolisms, ${ }^{\mathrm{f}}$ fatty acid metabolisms, ${ }^{9}$ glucose metabolisms, ${ }^{\mathrm{s}}$ sterol metabolisms

and HMG-CoA synthase 1 (Hmgcs1, an enzyme in cholesterol synthesis).

Not all the genes altered by the leptin receptor gene mutation were corrected by the metformin treatment. Among the 55 genes altered in vehicle-treated ZDF rats compared with Zucker lean rats, 19 (35\%) genes remained uncorrected in the metformin-treated ZDF rats. These included five genes involved in lipid metabolism (predominantly in fatty acid metabolism), one gene in glucose metabolism, and one gene in bile acid metabolism (Table 3).

Since degeneration and steatosis in hepatocytes were observed in metformin-treated ZDF rats, we examined the expression alterations of genes associated with possible cell damages such as inflammation, cell death, apoptosis, cell growth, fibrosis, and genes responsible for stresses (oxidative stress and endoplasmic reticulum stress). In the 38 genes which reached the criteria of fold change $\geq 2.0$ in the metformin-treated ZDF rats compared with Zucker lean rats, 23 genes (60.5\%) were annotated, among which, 1 gene was involved in cell cycle (Snf1lk, SNF1-like kinase), and 2 in cell growth (Igfbp1, and Socs2, namely suppressor of cytokine signaling 2). Moreover, no gene correlated with inflammation, cell death, apoptosis, fibrosis, oxidative stress or endoplasmic reticulum stress was found to have the expression fold change $\geq 2.0$, implying that long-term of 6 -month metformin treatment has no significant impact on the "bad" genes in ZDF rat liver.

\section{Validation of target genes}

Genes of interests were further validated by quantitative real-time PCR. We selected genes of the key enzymes involved in glucose (G6pdx) or lipid (Hmgcs1, Cyp7a1, Scd-1) metabolisms, and the gene with the most remarkable fold change (Igfbp1). Consistent with the microarray assay findings, gene expression levels of Igfbp1, Cyp7a1, Hmgcs1 and G6pdx were modified by the leptin receptor mutation and corrected by metformin treatment in the ZDF rats, whereas the expression level of Scd-1, an enzyme involved in fatty acid biosynthesis, was not corrected by the metformin treatment (Fig. 4).

\section{Discussion}

In this study, we investigated the therapeutic potential of metformin, a widely applied insulin sensitizer, in treating NAFLD. We performed unbiased transcriptomic analysis (microarray) in ZDF insulin resistance and NAFLD rat model to compare gene expression changes between groups treated with vehicle and relatively chronic metformin. Our study showed that 6-month treatment with metformin significantly lowered post-load blood glucose levels without affecting blood lipid profiles or the hepatocyte degeneration process. Metformin treatment showed the trend of alleviating the increase of liver enzyme levels,

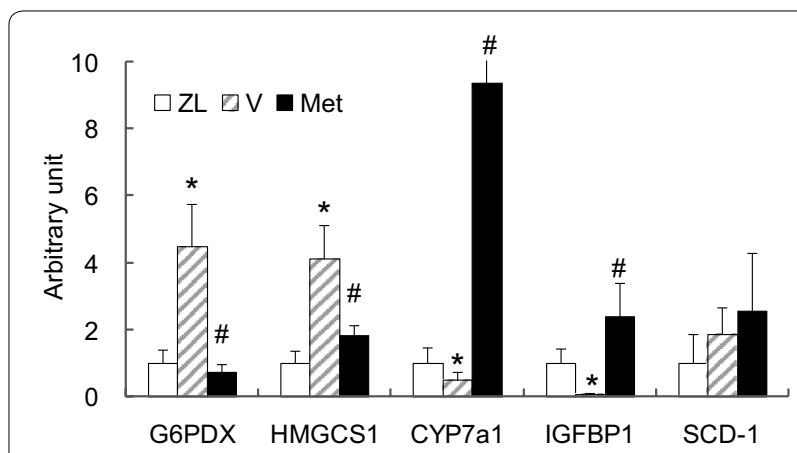

Fig. 4 Real-time PCR quantification of target genes. Total mRNA of liver tissues were extracted from Zucker lean rats (ZL), vehicle-treated ZDF rats $(V)$ and metformin-treated ZDF rats (Met) after 6-month treatment. Genes with interests were validated by quantitative real-time PCR. Similar to the results of microarray assay, gene expression levels of G6PDX, HMGCS1, CYP7a1 and IGFBP1were modified by leptin receptor mutation and corrected by metformin treatment, while the expression level of SCD-1 was not corrected by metformin treatment. Data are mean $\pm S D,{ }^{*} p<0.05, V$ versus $\mathrm{ZL}$; $\# p<0.05$, Met versus $V$ 
but this alleviation did not reach the statistical difference. Above that, as ZDF rats develop insulin resistance at a relatively early age (6-8 weeks), and the metformin treatment was started at the age of 8 weeks, our study also suggested that early interference with insulin resistance using metformin does not seem to prevent the development of liver steatosis. Clinical trials are rare to study the efficacy of insulin sensitizer in changing the risk of developing obesity-associated NAFLD, our study thus offered unique preclinical data for answering this important question. Another unexpected finding of this study was that most of the genes corrected by the metformin treatment were those involved in the metabolisms of glucose and cholesterol, but not of fatty acids. As lipid (both fatty acids and triglycerides) accumulation in the liver is an important initiation step in the development of NAFLD, it is, therefore, consistent with our finding that metformin treatment did not improve the liver histology in the ZDF model. Our data also suggest that metformin improved cholesterol metabolism (based on the gene expression changes), which is a topic insufficiently explored. Whether metformin protects liver cholesterol dysregulation or cholestasis represents an extremely interesting question that remains to be answered.

One important question in the NAFLD field is how NAFLD progresses to more severe stages of non-alcoholic steatohepatitis (NASH) or fibrosis. It is currently unclear whether metformin helps to protect NAFLD from developing NASH or fibrosis. Based on our data, we found that no genes associated with inflammation, apoptosis, fibrosis, or cell death were normalized in the metformin-treated ZDF rats. These data suggest metformin does not protect NAFLD from developing into more severe NASH or fibrotic stages, which requires further investigation.

Clinically, several studies have shown diabetic patients with fatty liver disease had improved serum aminotransferases level and insulin resistance after metformin treatment $[6,10-12]$. Therefore, metformin was used as a treatment candidate for NAFLD [3-6]. However, meta-analyses conclude that metformin therapy did not improve liver histology in patients with NAFLD or NASH $[19,20]$. In the latest AASLD guideline for NAFLD treatment, metformin is not recommended for treating NASH in adult patients [21].

It is notable that many genes affected by administration of metformin were overcorrected after the treatment, which implied the variation levels of most corrected genes are much greater than those in Zucker lean rats. For example, IGFBP1, which participates in multiple metabolic processes including lipid and glucose metabolism, showed down-regulation with fold change of 5.3 in vehicle-treated ZDF rats compared with that in Zucker lean rats, whereas it was 19.3-fold higher in metformin-treated ZDF rats compared with vehicle-treated ZDF rats. Consistently, other important lipid- and glucose-related enzymes (such as Cyp7a1, Sqle, Sds) showed similar expression profiles. Overcorrection of the metabolic genes by metformin might contribute to more severe hepatocyte injuries.

$\mathrm{CCO}$ is a component in the mitochondrial respiratory electron transport chain and functions in the generation of ATP derived from protons mainly produced during glucose and lipid catabolic processes. In this study, we demonstrated the overexpression of $\mathrm{CCO}$ protein and some genes related to glucose and cholesterol catabolic processes in ZDF rats treated with either vehicle or metformin. This overexpression may reflect a hyperfunctional state of mitochondria to compensate for the dysfunction in degenerated cells. Stronger CCO staining at the edge of the vacuoles and AMPK activation in the degenerated hepatocytes were found in the metformin-treated rats, which may reflect the hyperfunctional status of mitochondria with the intention to consume the cellular lipids. However, it is unclear whether metabolic overburden aggravates the pathological changes of the hepatocytes.

It should be borne in mind that the observations from animal models cannot be completely extrapolated to humans. In the ZDF rat model, leptin receptor gene mutation is a complicated scenario leading to rather severe hyperlipidemia. Therefore, the degenerated hepatocytes may not be replaced by new generation of hepatocytes under the background of severe hyperlipidemia and gene deficiency. In humans, however, these abnormalities may not exist. Thereafter, the leptin gene deficiency and severe hyperlipidemia might be responsible for the ineffectiveness of the long-term metformin treatment on fatty liver in Zucker rats. In addition, the sample size of this study is relatively small, which might be a limitation to show a protective effect of metformin on liver enzymes. Further experiments and larger sample size can produce more reliable results.

\begin{abstract}
Abbreviations
ALT: alanine aminotransferase; AST: aspartate transaminase; CCO: cytochrome c oxidase; Cyp7a1: cytochrome P450, family 7, subfamily a, polypeptide 1; G6pdx: glucose-6-phosphate dehydrogenase; IGFBP1: insulin-like growth factor-binding protein-1; NAFLD: non-alcoholic fatty liver disease; NASH: non-alcoholic steatohepatitis; OGTT: oral glucose tolerance tests; ZDF: Zucker diabetic fatty.
\end{abstract}

\section{Acknowledgements \\ Not applicable.}

\section{Authors' contributions}

YS, XK, RF, SF, and TY made contributions to experiment design and manuscript writing. YY, HM, SZ, WL, and PR made contributions to experimental operation. All authors read and approved the final manuscript. 


\section{Funding}

The work is partially supported by a grant from the National Natural Science Foundation of China (Grant nos. 81660150 and 81572518), and Natural Science Foundation of Guangdong Province (Grant no. 2017A030313792).

\section{Availability of data and materials}

The datasets used and/or analyzed during the current study are available from the corresponding author on reasonable request.

\section{Ethics approval and consent to participate}

Ethical approval for animal studies was according to the Animal Experimentation Ethics Committee of The Fudan University Pudong Medical Center.

\section{Consent for publication}

Not applicable.

\section{Competing interests}

The authors declare that they have no competing interests.

\section{Author details}

${ }^{1}$ Department of Clinical Nutrition, The First Affiliated Hospital, Sun Yat-sen University, Guangzhou 510080, China. ${ }^{2}$ Endoscopy Center, The Sixth Affiliated Hospital, Sun Yat-sen University, Guangzhou 510655, China. ${ }^{3}$ Department of Biosciences and Nutrition, Karolinska Institute, Stockholm, Sweden. ${ }^{4}$ Center for Medical Research and Innovation, Shanghai Pudong Hospital, Fudan University Pudong Medical Center, Shanghai 201399, China.

Received: 20 August 2019 Accepted: 4 November 2019 Published online: 12 November 2019

\section{References}

1. Pentikainen PJ, Voutilainen E, Aro A, Uusitupa M, Penttila I, Vapaatalo H. Cholesterol lowering effect of metformin in combined hyperlipidemia: placebo controlled double blind trial. Ann Med. 1990;22(5):307-12.

2. Wang N, Zhang J, Wu Y, Liu J, Liu L, Guo X. Metformin improves lipid metabolism disorders through reducing the expression of microsomal triglyceride transfer protein in OLETF rats. Diabetes Res Clin Pract. 2016;122:170-8.

3. Feng W, Gao C, Bi Y, Wu M, Li P, Shen S, et al. Randomized trial comparing the effects of gliclazide, liraglutide, and metformin on diabetes with nonalcoholic fatty liver disease. J Diabetes. 2017;9(8):800-9.

4. Garcia Diaz E, Guagnozzi D, Gutierrez V, Mendoza C, Maza C, Larranaga Y, et al. Effect of incretin therapies compared to pioglitazone and gliclazide in non-alcoholic fatty liver disease in diabetic patients not controlled on metformin alone: an observational, pilot study. Endocrinol Nutr. 2016;63(5):194-201.

5. Haukeland JW, Konopski Z, Eggesbo HB, von Volkmann HL, Raschpichler G, Bjoro K, et al. Metformin in patients with non-alcoholic fatty liver disease: a randomized, controlled trial. Scand J Gastroenterol. 2009;44(7):853-60.

6. Loomba R, Lutchman G, Kleiner DE, Ricks M, Feld JJ, Borg BB, et al. Clinical trial: pilot study of metformin for the treatment of non-alcoholic steatohepatitis. Aliment Pharmacol Ther. 2009;29(2):172-82.

7. Madsen A, Bozickovic O, Bjune Jl, Mellgren G, Sagen JV. Metformin inhibits hepatocellular glucose, lipid and cholesterol biosynthetic pathways by transcriptionally suppressing steroid receptor coactivator 2 (SRC-2). Sci Rep. 2015;5:16430.

8. Wulffele MG, Kooy A, de Zeeuw D, Stehouwer CD, Gansevoort RT. The effect of metformin on blood pressure, plasma cholesterol and triglycerides in type 2 diabetes mellitus: a systematic review. J Intern Med. 2004;256(1):1-14

9. Tamura Y, Watada H, Sato F, Kumashiro N, Sakurai Y, Hirose T, et al. Effects of metformin on peripheral insulin sensitivity and intracellular lipid contents in muscle and liver of overweight Japanese subjects. Diab Obes Metab. 2008;10(9):733-8.

10. Bugianesi E, Gentilcore E, Manini R, Natale S, Vanni E, Villanova N, et al. A randomized controlled trial of metformin versus vitamin $E$ or prescriptive diet in nonalcoholic fatty liver disease. Am J Gastroenterol. 2005;100(5):1082-90.

11. Idilman R, Mizrak D, Corapcioglu D, Bektas M, Doganay B, Sayki M, et al. Clinical trial: insulin-sensitizing agents may reduce consequences of insulin resistance in individuals with non-alcoholic steatohepatitis. Aliment Pharmacol Ther. 2008;28(2):200-8.

12. Shields WW, Thompson KE, Grice GA, Harrison SA, Coyle WJ. The effect of metformin and standard therapy versus standard therapy alone in nondiabetic patients with insulin resistance and nonalcoholic steatohepatitis (NASH): a pilot trial. Therap Adv Gastroenterol. 2009;2(3):157-63.

13. Cone CJ, Bachyrycz AM, Murata GH. Hepatotoxicity associated with metformin therapy in treatment of type 2 diabetes mellitus with nonalcoholic fatty liver disease. Ann Pharmacother. 2010;44(10):1655-9.

14. Hashmi T. Probable hepatotoxicity associated with the use of metformin in type 2 diabetes. BMJ Case Rep. 2011. https://doi.org/10.1136/ bcr.04.2011.4092.

15. Miralles-Linares F, Puerta-Fernandez S, Bernal-Lopez MR, Tinahones FJ, Andrade RJ, Gomez-Huelgas R. Metformin-induced hepatotoxicity. Diabetes Care. 2012;35(3):e21.

16. Kutoh E. Possible metformin-induced hepatotoxicity. Am J Geriatr Pharmacother. 2005;3(4):270-3.

17. Desilets DJ, Shorr AF, Moran KA, Holtzmuller KC. Cholestatic jaundice associated with the use of metformin. Am J Gastroenterol. 2001;96(7):2257-8.

18. Zhou G, Myers R, Li Y, Chen Y, Shen X, Fenyk-Melody J, et al. Role of AMP-activated protein kinase in mechanism of metformin action. J Clin Investig. 2001;108(8):1167-74.

19. Rakoski MO, Singal AG, Rogers MA, Conjeevaram H. Meta-analysis: insulin sensitizers for the treatment of non-alcoholic steatohepatitis. Aliment Pharmacol Ther. 2010;32(10):1211-21.

20. Musso G, Gambino R, Cassader M, Pagano G. A meta-analysis of randomized trials for the treatment of nonalcoholic fatty liver disease. Hepatology. 2010;52(1):79-104.

21. Chalasani N, Younossi Z, Lavine JE, Charlton M, Cusi K, Rinella M, et al. The diagnosis and management of nonalcoholic fatty liver disease: practice guidance from the American Association for the Study of Liver Diseases. Hepatology. 2018;67(1):328-57.

\section{Publisher's Note}

Springer Nature remains neutral with regard to jurisdictional claims in published maps and institutional affiliations.

Ready to submit your research? Choose BMC and benefit from:

- fast, convenient online submission

- thorough peer review by experienced researchers in your field

- rapid publication on acceptance

- support for research data, including large and complex data types

- gold Open Access which fosters wider collaboration and increased citations

- maximum visibility for your research: over $100 \mathrm{M}$ website views per year

At BMC, research is always in progress.

Learn more biomedcentral.com/submissions 